\title{
Construcción de una praxeología para la enseñanza en la institución de formación del profesorado
}

\author{
Construction of a praxeology for teaching \\ in the teacher training institution
}

\author{
Alicia Ruiz-Olarría ${ }^{1}$ \\ Marianna Bosch Casabò ${ }^{2}$ \\ Josep Gascón Pérez ${ }^{3}$
}

\begin{abstract}
Resumen: Presentamos una metodología para la reconstrucción de praxeologías matemáticas para la enseñanza basada en los recorridos de estudio e investigación para la formación del profesorado. Ejemplificamos dicha metodología mediante la construcción de una praxeología para la enseñanza, que surge como respuesta a la cuestión generatriz: "¿Cómo organizar la enseñanza de la modelización funcional elemental en la Enseñanza Secundaria Obligatoria (12-16 años) y qué papel asignar a la proporcionalidad en dicha organización?". La experimentación se ha llevado a cabo en el Máster oficial de formación del profesorado de secundaria.
\end{abstract}

Palabras clave: REI, TAD, praxeología, modelización matemática, formación de profesorado de matemáticas.

Abstract: We present a methodology for the reconstruction of a mathematical praxeology for teaching based on study and research paths for teacher

Fecha de recepción: 26 de febrero de 2019. Fecha de aceptación: 1 de julio de 2019.

1 Facultad de Formación del Profesorado y Educación. Departamento de Didácticas Específicas. Universidad Autónoma de Madrid, España. alicia.ruiz@uam.es, orcid.org/ 0000-0003-1552-1434

2 IQS. Universitat Ramon Llull, España. marianna.bosch@iqs.url.edu, orcid.org/ 0000-0001-9756-116X

3 Facultad de Ciencias. Departamento de Matemáticas. Universidad Autónoma de Barcelona, España. gascon@matuab.cat, orcid.org/ 0000-0001-5570-1144 
education. This methodology is illustrated through the construction of a praxeology for teaching that emerges as an answer to the generating question: "How to organize the teaching of about elementary functional modeling at lower secondary school (12-16 years old) and what role can be assigned to proportionality in this organization?" The experimentation took place in the Master for secondary school teacher education.

Keywords: SRP, ATD, praxeologie, mathematical modeling, teacher training in mathematics.

\section{INTRODUCCIÓN: EL OFICIO Y LA PROFESIÓN DOCENTE}

La teoría antropológica de lo didáctico (TAD) ha estado siempre estrechamente relacionada con la formación inicial y continua de los profesores por distintas razones. En primer lugar, porque numerosos profesores forman parte de diversos equipos de investigación que trabajan en el ámbito de la TAD. En segundo lugar, desde el momento en que Chevallard (1985) evidencia el fenómeno de la transposición didáctica, la TAD fue uno de los primeros enfoques en considerar como objeto de estudio e investigación, no sólo las actividades de enseñanza y aprendizaje en el aula, sino todo el proceso que va desde la creación y utilización del saber matemático hasta su incorporación en la escuela como saber enseñado. Dicho objeto de estudio incluye, además, todas las instituciones que participan en este proceso entre las que se cuentan el propio profesorado y aquellas que intervienen en su formación inicial y continua. En tercer lugar, porque algunos investigadores que trabajan en el marco de la TAD se han visto involucrados en la formación del profesorado de los distintos niveles educativos. De ahí, que el desarrollo de esta teoría se haya visto potenciado por los problemas que surgen en dichos procesos de formación y el esfuerzo por aportar elementos de respuesta. No sorprende, pues, que la formación de profesores se considere como uno de los principales ámbitos de estudio e investigación de la TAD.

Por otra parte, aun hoy persiste en amplios sectores de la sociedad -incluida la "noosfera"-, una concepción del oficio docente que conduce a responsabilizar al profesor de los resultados de la enseñanza, puesto que es visto como un pequeño productor independiente que se debe procurar sus recursos de manera individual. En consecuencia, el profesor se ve abocado a considerar que los 
problemas y dificultades que encuentra en el desarrollo de su profesión provienen, principalmente, de sus limitaciones personales. Si el docente se viera a sí mismo como miembro de una profesión, su oficio cambiaría profundamente al poder identificar varios de los problemas docentes no como limitaciones propias sino como problemas de la profesión. De esta forma, la responsabilidad de buscar respuestas no recaería sobre el profesor, individualmente considerado, sino sobre la profesión como institución, que a su vez debería liderar el cambio en las creencias más arraigadas acerca del oficio docente.

\section{EL PROBLEMA DE LA FORMACIÓN DEL PROFESORADO EN LA TAD: LOS RECORRIDOS DE ESTUDIO E INVESTIGACIÓN PARA LA FORMACIÓN DEL PROFESORADO}

Desde la TAD se propone formular el problema de la formación del profesorado en términos de las praxeologías por enseñar, las praxeologías para la enseñanza y las praxeologías para la profesión, como propone Cirade (2006), con la consideración de lo siguiente:

¿Cuáles son las cuestiones cruciales que deben afrontar los profesores en su práctica docente y qué puede hacer la formación para ayudarlos a construir respuestas satisfactorias -en forma de praxeologías- a estas cuestiones? En particular, ¿cómo se generan las cuestiones que están en el origen de las praxeologías matemáticas por enseñar y de las praxeologías matemáticas para la enseñanza? ¿Cuáles son los problemas que constituyen la razón de ser de las praxeologías de la profesión docente?

Si la propuesta del dispositivo didáctico para la enseñanza y el aprendizaje de las matemáticas se formula mediante los recorridos de estudio e investigación (REI), con el fin de integrar la razón de ser de los saberes escolares en el corazón del proceso de estudio (Chevallard, 2013), y favorecer el desarrollo de las condiciones que se requieren para hacer posible una actividad matemática funcional, como lo han señalado Barquero (2009), Ruiz-Munzón (2010) y Serrano (2013), parece evidente que una aproximación progresiva al nuevo paradigma del cuestionamiento del mundo requiera la transformación de las condiciones del trabajo matemático del profesorado y, en particular, un replanteamiento radical del tipo de formación necesaria para la enseñanza de las matemáticas. 
Así, el dispositivo para la formación del profesorado que se propone desde la TAD tiene estructura de recorrido de estudio e investigación (REI-FP), recorrido que parte de una cuestión problemática para la profesión docente, que denominamos $Q_{0}$-FP. El estudio de esta cuestión se va a articular en cinco módulos que constituyen los componentes del REI-FP, con las características que se describen a continuación.

\subsection{Módulo $\mathrm{M}_{0}$ : ¿Cómo ENSEÑAR UN CONTENIDO C?}

A fin de partir de una cuestión inicial que forme parte de la problemática de la profesión, los REI-FP tienen origen en un determinado ámbito, competencia o contenido matemático C -la proporcionalidad, el álgebra elemental, la modelización, etc.- y en un tipo de cuestión que es umbilical para la profesión: "¿Cómo organizar el estudio de C?"

Este módulo contiene tanto la construcción de $Q_{0}$-FP (su elección y asunción), así como las exploraciones iniciales con vista a elaborar primeros elementos de respuesta, generalmente a partir de los media más habituales para los profesores: currículum, libros de texto, revistas para el profesorado, revistas de investigación, centros de recursos, webs, etc. El rol de los formadores en este proceso no es el de aportar elementos de respuesta para darlos a conocer a los profesores, sino guiarlos en la búsqueda de estos elementos y, sobre todo, iniciarlos en la práctica de los gestos básicos del cuestionamiento didáctico:

¿Qué es $C$ ? ¿De dónde viene? ¿En qué ámbitos matemáticos y no matemáticos se utiliza o utilizaba? ¿Por qué hay que enseñarlo? ¿Cuáles son sus razones de ser en la matemática escolar (las establecidas explícita o implícitamente y las potenciales)? ¿Qué propuestas de enseñanza existen? ¿Qué se dice o sabe de ellas?, etc.

Es muy importante que durante todo el REI-FP, estas cuestiones se mantengan vivas, es decir, a la vez presentes y sin respuesta definitiva. Desafortunadamente, no es la actitud habitual en nuestra sociedad, ni en relación con las cuestiones docentes ni en relación con muchas otras. Y. Chevallard (2003) retomaba al respecto algunas afirmaciones totalmente pertinentes del literato francés Maurice Blanchot: ${ }^{4}$

4 Cita traducida por autores. 
Tendemos a ver el mundo como un conjunto de respuestas a preguntas que pronto olvidaremos. "En los llamados períodos felices, solo las respuestas parecen estar vivas", observa con razón Maurice Blanchot (1907-2003). Por el contrario, ver el mundo como un conjunto de preguntas -posiblemente sin (buenas) respuestas- no sucede espontáneamente [...] Además, la asunción de una pregunta Q puede reducirse a la nada por la imposición demasiado rápida, intempestiva, de la respuesta dogmática R que se adelantaría solamente para ocultar y más tarde olvidar la pregunta, según un mecanismo que Blanchot ha condensado en un valioso aforismo: "La respuesta es el infortunio de la cuestión." Sea como sea, la respuesta R debe considerarse como provisional, un simple medio de satisfacer necesidades perentorias, en un gesto que no es fijo ni cerrado sobre sí mismo, aunque lo provisional pueda parecer durable. Cualquier respuesta a una pregunta debe considerarse como puesta a disposición para su aprovechamiento fértil en forma de deconstrucciones y reconstrucciones: "cuando afirmas, no dejas de cuestionar", enfatizó Blanchot. (Chevallard, 2003, p.7)

Podemos, en definitiva, definir este módulo inicial y transversal del REI-FP como el módulo de la problemática del profesor, donde se parte de una cuestión o de un conjunto de cuestiones propias de la profesión para las que se irán elaborando poco a poco elementos de respuesta colectivos y para las que, al final del proceso, cada uno de los profesores deberá también poder aportar sus respuestas personales.

Estas cuestiones están en la base de la investigación didáctica ya que tienen que ver con "las razones" que justifican el estudio de un objeto matemático o de un ámbito de la matemática. Por otra parte, y dado que se puede considerar este módulo inicial también trasversal, se espera su enriquecimiento con algunas otras surgidas a lo largo del estudio en los módulos siguientes.

\subsection{MÓdULO $\mathrm{M}_{1}$ : VIVIR UN REI}

En el transcurso del módulo $\mathrm{M}_{0}$ se espera que aparezca una variedad de respuestas institucionales elaboradas en distintos ámbitos, como propuestas de enseñanza de $C$. Puesto que no es fácil obtener elementos cuestionadores o refundadores de $C$, la herramienta principal disponible en didáctica para este cuestionamiento es el modelo epistemológico de referencia (MER) ${ }^{5}$-relativo a $C$ - y las distintas formas

\footnotetext{
5 Un MER es una interpretación, en términos praxeológicos, de cierto ámbito de la actividad matemática.
} 
que pueda tomar. Dado que este MER puede sustentar la puesta en marcha de un REl, una posible manera de poner el MER a disposición de los profesores es realizar el REI directamente durante el proceso de formación.

En consecuencia, el objetivo de este módulo es que el estudiante-profesor viva un REl como miembro del sistema didáctico $S\left(X, Y, Q_{1}\right)$ situado en la institución de formación del profesorado, donde $X$ denota la comunidad de estudiantes para profesor, $Y$ los formadores -aquí como directores del estudio- y $Q_{1}$ la cuestión a la que deberán aportar su propia respuesta. En esta fase del recorrido de formación, $Q_{1}$ es la cuestión generadora del REI llevado a cabo en secundaria.

\subsection{MÓdulo $\mathrm{M}_{2}$ : "ANALIZAR EL REI VIVIDO"}

La cuestión generatriz $Q_{2}$ que dirige esta segunda fase del recorrido de formación debe girar en torno al cuestionamiento matemático-didáctico del REI vivido anteriormente en posición de estudiante.

En este módulo se debe cuestionar y analizar en profundidad la estructura y la dinámica del REI vivido, tanto en lo que respecta a las praxeologías matemáticas construidas efectivamente, como a la organización didáctica de este proceso, en términos de la articulación de los momentos y las dialécticas del estudio, de los gestos y las técnicas y tecnologías didácticas que se han puesto en juego, y en términos de las responsabilidades que han asumido los profesores en formación en su papel de estudiantes y el formador en su papel de director del proceso de estudio (Chevallard, 1999). Para llevar a cabo este análisis, los profesores en formación pueden recurrir al material empírico utilizado en el módulo $\mathrm{M}_{0}$, a los materiales que describen la experimentación realizada previamente con los alumnos de Secundaria ${ }^{6}$, así como al material elaborado en su propia vivencia del REI.

Es obvio que, como en todo análisis científico, la elección de las cuestiones que dirigirán el análisis estará guiada por el punto de vista que se tome para realizarlo. En este caso, al igual que en el módulo anterior, el punto de vista lo proporcionan el MER construido en la investigación y los distintos elementos de análisis de los procesos de estudio que proporciona la TAD. EI MER muestra las limitaciones del modelo epistemológico dominante en la institución de

6 En muchas ocasiones el REl vivido por los profesores en formación ha sido previamente experimentado con alumnos de Secundaria. 
enseñanza (infantil, primaria, secundaria, ...) y una manera de hacerlo asequible a los profesores en formación, es mediante las cuestiones que de manera explícita se plantean en este módulo.

\subsection{MÓdULO $\mathrm{M}_{3}$ : DISEÑAR UN REI}

La cuestión generatriz $Q_{3}$ que dirige esta fase del recorrido de formación puede enunciarse así:

¿Cómo llevar a cabo la tarea de diseñar un REI para los alumnos de cierta etapa educativa, análogo al vivido, y analizado en las fases anteriores?

Esta cuestión da origen a otras más concretas tales como: (a) ¿qué elementos componen el diseño de un REl?, (b) ¿cuál es el orden más razonable para diseñar cada uno de dichos elementos?, y (c) ¿cómo deben expresarse materialmente dichos elementos del REl?

Los criterios básicos para dar respuesta a estas cuestiones y explicitar un diseño didáctico a priori de un REl análogo al vivido surgen en cierta medida de las respuestas aportadas en los módulos anteriores, teniendo en cuenta que la aplicación de los criterios matemático-didácticos obtenidos no es inmediata.

\subsection{MÓdULO $\mathrm{M}_{4}$ : GESTIONAR Y EXPERIMENTAR UN REI}

El objetivo de este módulo es doble. Por un lado, dar soporte regular a los profesores que se inician en el diseño y gestión de los REl y, por otro, recoger las cuestiones, dificultades y obstáculos que han podido surgir durante esta gestión. Este trabajo experimental debería proporcionar criterios fundados para modificar el diseño de los REI de cara a nuevas experimentaciones e introducir a los profesores a la problemática ecológica en didáctica, mediante el análisis de las condiciones y restricciones institucionales con que se van a topar.

Aunque todavía no podemos decir gran cosa de este módulo, puesto que son pocas las experimentaciones realizadas (Barquero, Bosch y Romo, 2015, 2018; Florensa, Bosch, y Gascón, 2016), consideramos que es clave por diversos motivos. En primer lugar, porque constituye un ámbito de validación experimental final del REI-FP llevado a cabo y de las nuevas posibles formas de organizar 
tanto los contenidos matemáticos curriculares como su enseñanza y aprendizaje. En segundo lugar, porque es también una fuente de nuevas cuestiones para proseguir con el estudio, tanto para los profesores que lo experimentan como para los investigadores o formadores. Finalmente, creemos que este módulo puede representar un buen terreno de cooperación entre profesores e investigadores en una perspectiva análoga a la que propone el equipo de la Universidad de Turín dirigido por Arzarello et al. (2013).

\section{DISEÑO Y DESARROLLO DE UN REI-FP EN TORNO AL PROBLEMA DE LA INTEGRACIÓN DE LA PROPORCIONALIDAD EN LA MODELIZACIÓN FUNCIONAL ELEMENTAL DE LA ENSEÑANZA SECUNDARIA OBLIGATORIA}

Al plantearse la cuestión de la formación del profesorado, parece razonable construir las praxeologías para la enseñanza sobre la base de las praxeologías matemáticas por enseñar reconstruidas y descritas por el correspondiente MER alternativo -el modelo construido en la investigación didáctica-, en lugar de hacerlo a partir de las organizaciones matemáticas escolares habituales. En coherencia con ello, parece obvio utilizar algunos de los REI diseñados y experimentados previamente en la enseñanza secundaria. Asimismo, es necesario delimitar el ámbito institucional donde se situará el problema relativo a la reconstrucción de praxeologías para la enseñanza.

En la investigación llevada a cabo (Ruiz-Olarría, 2015), nos hemos centrado en la formación inicial del profesorado y, más concretamente, en la formación matemático-didáctica que es posible impartir en los estudios del máster oficial (en España) de formación del profesorado de secundaria. Uno de los problemas abordados en esta investigación ha sido el de la construcción y reconstrucción de una praxeología para la enseñanza mediante la puesta en marcha (diseño y gestión didáctica) de los módulos $\mathrm{M}_{0}, \mathrm{M}_{1}$ y $\mathrm{M}_{2}$ del dispositivo REl-FP.

Una vez constatados los problemas didácticos asociados al fenómeno de la desarticulación y la pérdida de sentido de la modelización funcional en la educación secundaria obligatoria (ESO) y, muy especialmente, para responder al problema docente en torno a la proporcionalidad, García (2005) y García, Gascón, Ruiz Higueras y Bosch (2006) proponen un MER que sitúa la razón de ser de la proporcionalidad en el ámbito de las modelizaciones funcionales elementales. En concreto, el MER propuesto, parte de la caracterización de diferentes tipos de variación de magnitudes. Para ello, y teniendo en cuenta las restricciones 
institucionales que provienen de la ESO, se consideran dos magnitudes $\mathrm{M}$ y $\mathrm{M}^{\prime}$ discretas, se parte de un conjunto de cantidades de la primera magnitud que están en progresión aritmética y se analiza el tipo de variación de las cantidades correspondientes de la segunda magnitud.

En este punto aparece la necesidad de tomar una decisión que será fundamental en la construcción del MER: ¿Qué criterio utilizar para definir diferentes "tipos de variación"? En la práctica se utiliza el criterio que proporciona la imposición de determinadas condiciones elementales sobre el tipo de variación. Por ejemplo, se define la condición de equidad imponiendo que las cantidades correspondientes de la magnitud $\mathrm{M}^{\prime}$ también estén en progresión aritmética. Y, por extensión, la condición de diferencias constantes de orden $n$ imponiendo que las diferencias de orden $n$ de la sucesión de imágenes sean constantes.

De esta forma, la relación de proporcionalidad directa queda caracterizada como un caso particular de la condición de equidad. En efecto, la relación de proporcionalidad directa cumple la condición de equidad y, además, transforma progresiones geométricas en progresiones geométricas de la misma razón, lo que comporta que cumpla la condición de linealidad. Junto a ella aparecen otros tipos de relaciones (afines, cuadráticas, exponenciales, de proporcionalidad inversa, etc.) según el tipo de variación que caracteriza cada tipo de relación.

En definitiva, el MER propuesto en García (2005) integra, mediante la modelización funcional elemental, el estudio de diferentes tipos de sistemas en los que las cantidades de magnitud son susceptibles de variar según las condiciones enunciadas, conformando una organización matemática regional articulada en torno a la teoría de las funciones reales de variable real.

Igualmente, en este mismo trabajo se describe con todo detalle el diseño a priori y la experimentación del REI llevado a cabo con alumnos de la ESO y sustentado en el MER que éste propone, sobre los que nos hemos apoyado para el diseño y la experimentación de los módulos $M_{0}, M_{1}$ y $M_{2}$ del REl-FP. En Ruiz-Olarría (2015), se describe con detalle el trabajo relativo al análisis y experimentación de un REI-FP en torno a la proporcionalidad y su integración en la modelización funcional elemental, por lo que en este trabajo nos limitaremos a describir una estrategia encaminada a reconstruir praxeologías matemáticas para la enseñanza, lo que consideramos como uno de los principales frutos de la puesta en marcha de un REI-FP. A continuación, presentamos un esquema (véase figura 1) de las etapas realizadas en la construcción de una praxeología para la enseñanza que se describirán con detalle en la sección 4. 
Figura 1. Esquema parcial de la metodología para construir praxeologías para la enseñanza.

(1) «Construcción» de un fenómeno didáctico en torno a cierto ámbito $\mathrm{A}_{\mathrm{M}}$ de la actividad matemática

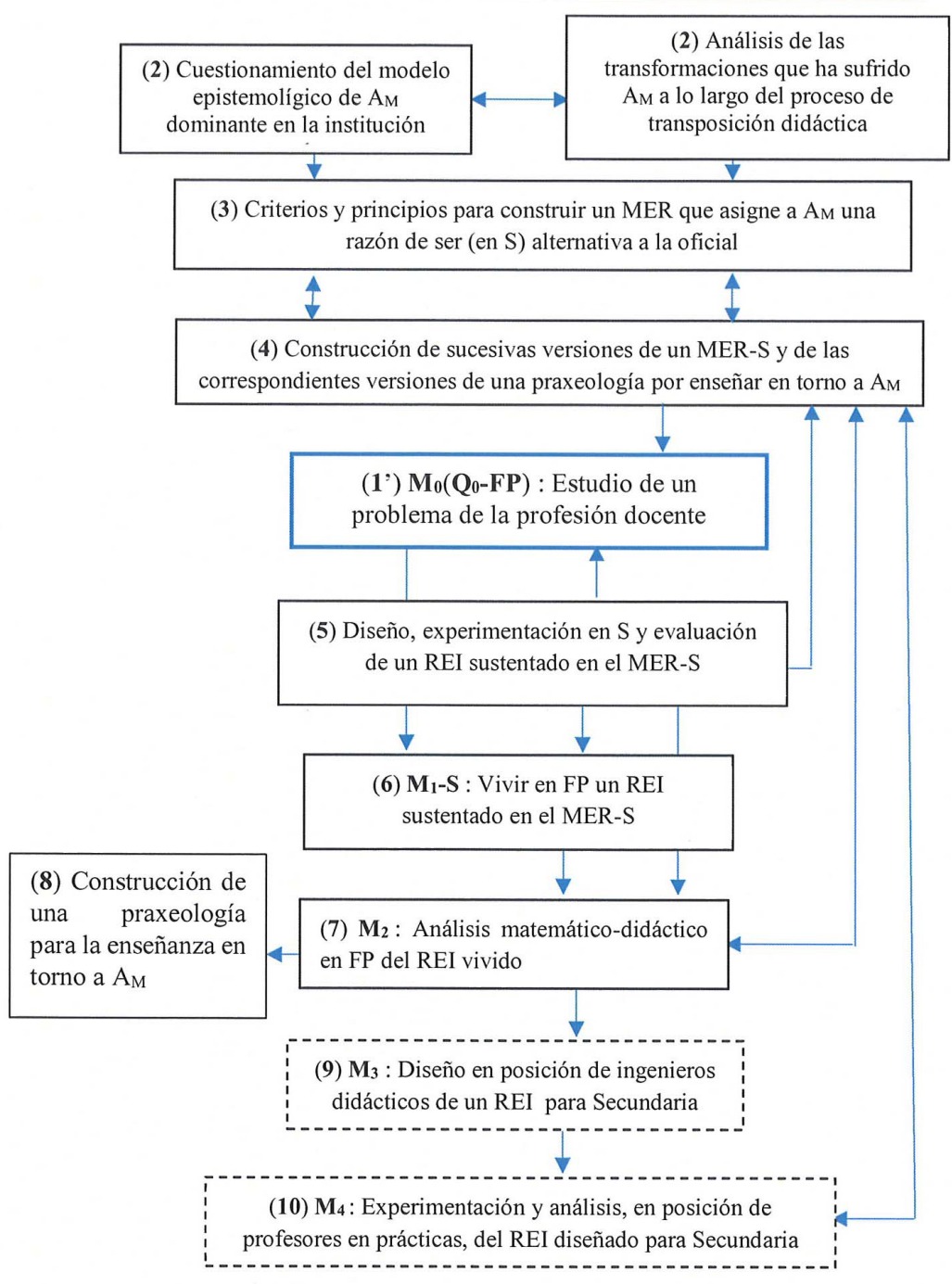




\section{ESTRATEGIA METODOLÓGICA PARA RECONSTRUIR UNA PRAXEOLOGÍA PARA LA ENSEÑANZA}

Como hemos indicado, en Ruiz-Olarría (2015) se describe la estrategia llevada a cabo en un Máster de Formación del Profesorado de Secundaria para reconstruir una versión de una praxeología matemática para la enseñanza en torno a la modelización funcional elemental (MFE), mediante la experimentación de los Módulos $M_{0}, M_{1}$ y $M_{2}$ de un REI-FP.

Dado que la estructura de esta estrategia es aplicable a otros muchos casos, con el fin de reconstruir praxeologías matemáticas para la enseñanza (Licera, 2017), consideramos que la citada estrategia constituye una aportación central en la metodología que propone la TAD para la formación del profesorado. Resumiremos brevemente en lo que sigue sus principales etapas y las relaciones entre ellas, advirtiendo que no es posible establecer una linealidad temporal estricta entre las mismas, puesto que algunas se desarrollan de manera simultánea en la práctica efectiva y, en ciertos casos, se establecen relaciones recíprocas entre etapas debido a que el desarrollo de cada una de ellas provoca modificaciones importantes en el desarrollo de las otras.

Señalaremos cada una de estas etapas con el mismo número que aparece en el esquema adjunto (ver figura 1).

(1) y (1') Dialéctica entre la formulación de un problema de la profesión docente y la toma en consideración de un fenómeno didáctico

La estrategia puesta en marcha parte de la constatación de un problema de la profesión docente que, en nuestro caso, es el problema relativo al diseño y gestión, en la ESO, de la enseñanza y el aprendizaje de la MFE, incluyendo el papel que juega la relación de proporcionalidad en la matemática escolar (Ruiz-Olarría, 2015). Simultáneamente, dicha estrategia se inicia con la toma en consideración de un fenómeno didáctico emergente en dicho ámbito que se manifiesta en la ausencia de problematización de los sistemas de variación. En la organización matemática escolar, éstos se utilizan de forma transparente y accesoria, lo que permite explicar en parte el aislamiento de la proporcionalidad del resto de relaciones funcionales y las dificultades del currículo (y, por lo tanto, de los profesores) para integrar el estudio de la proporcionalidad en una praxeología regional en torno a la MFE. Esta simultaneidad entre la constatación de un problema de la profesión docente relativo a un ámbito de la actividad 
matemática escolar, y la toma en consideración de un fenómeno didáctico emergente en dicho ámbito no es meramente una coincidencia temporal. De hecho, la constatación de un problema docente constituye un componente importante de la base empírica para construir el fenómeno didáctico y, recíprocamente, la toma en consideración del fenómeno en cuestión permite replantear el problema docente como un verdadero problema de investigación didáctica.

En nuestro caso, la formulación del problema de la profesión docente se planteó en el módulo $\mathrm{M}_{0}$ en los términos siguientes:

$\mathrm{Q}_{0}$-FP: ¿Cómo organizar la enseñanza de la MFE en la ESO y qué papel asignar a la proporcionalidad en dicha organización?

(2) Cuestionamiento de la matemática escolar, análisis de los procesos transpositivos y caracterización del modelo epistemológico dominante en Secundaria

La formulación del fenómeno didáctico citado -ausencia de problematización de los sistemas de variación- y el replanteamiento del problema docente asociado requieren el cuestionamiento de la organización matemática escolar en torno a la MFE y, correlativamente, el análisis de las transformaciones que ha sufrido dicho ámbito a lo largo del proceso de transposición didáctica, para poder caracterizar el modelo epistemológico dominante en la ESO en torno a la MFE. Para llevar a cabo dicho cuestionamiento, la estrategia metodológica requiere que se planteen cuestiones tales como:

¿Qué se entiende en dicha institución por MFE? ¿Cuál es la razón de ser "oficial" que la institución escolar le asigna? ¿Qué actividades matemáticas se llevan a cabo en la ESO en las que intervenga la MFE? ¿Cómo se relaciona la proporcionalidad con el resto de las relaciones funcionales elementales que aparecen en la matemática escolar?

En resumen, el objetivo de este cuestionamiento y del correspondiente análisis transpositivo, consiste en indagar el sistema de reglas y principios que regulan la estructura y el funcionamiento de la MFE en el sistema escolar y su relación con la proporcionalidad, esto es, analizar la "economía escolar" (Josep Gascón, 2011) de dicho ámbito de la actividad matemática en la ESO. 


\section{(3) Criterios y principios para construir un MER-S}

El análisis del citado modelo epistemológico dominante en la ESO en torno a la MFE y la clarificación de la consiguiente "razón de ser oficial" que se asigna a dicho ámbito en la matemática escolar aportan, con la ayuda de los instrumentos que proporciona la TAD, algunos criterios y principios necesarios para construir un MER-S, esto es, un MER que asigne en la enseñanza secundaria una razón de ser alternativa (o, según el caso, complementaria) a la razón de ser oficial. Estos criterios y principios se basan esencialmente en el análisis del fenómeno didáctico y, correlativamente, en el estudio del problema de la profesión docente asociado, por lo que su formulación reflejará los nuevos objetivos matemático-didácticos que el MER-S encarnará. Esta explicitación de objetivos matemático-didácticos posibles constituye una de las etapas cruciales de la estrategia metodológica.

En nuestro caso, la nueva praxeología por enseñar redefinida por el MER-S explicita como objetivos didáctico-matemáticos posibles la integración de la problematización de los sistemas de variación, la superación del aislamiento de la proporcionalidad del resto de relaciones funcionales y su integración en una praxeología regional en torno a la MFE.

(4) Construcción de un MER-S y de la correspondiente praxeología matemática por enseñar en Secundaria

La estrategia metodológica continúa con la construcción efectiva de una primera versión del MER-S a partir de los citados principios y criterios, lo que comporta una nueva redefinición de lo que se entiende en la ESO por "modelización funcional elemental", de su relación con la proporcionalidad y de su posición curricular con respecto al resto de áreas de la matemática escolar. En consecuencia, cada versión del MER-S delimita, reestructura y redefine, una praxeología por enseñar en torno a la MFE en la ESO (que puede diferir ampliamente de la praxeología por enseñar oficial). Se supone que esta nueva praxeología por enseñar permitirá llevar a cabo un proceso de estudio que se acerque progresivamente (a medida que se construyen nuevas versiones de la misma) a ciertos objetivos matemático-didácticos determinados de antemano -como así se indica en el punto (3)- y, potencialmente diferentes a los que era posible alcanzar en el ámbito de la actividad matemática escolar -en el caso que nos ocupa, en torno a la MFE, cuando estaba definida y estructurada sobre la base 
del antiguo modelo epistemológico dominante-. La construcción efectiva del MER-S puede provocar cambios en los criterios y principios que regulan a priori su construcción.

\section{(5) Diseño, experimentación y evaluación de un REl en Secundaria}

Una vez construida en el ámbito de la investigación didáctica una versión del MER-S que, no debe olvidarse, tiene el estatus de hipótesis científica, la estrategia metodológica que estamos describiendo -cuyo objetivo final es reconstruir una praxeología para la enseñanza- continúa con el diseño, experimentación y evaluación en secundaria de un REI sustentado en dicho MER-S. Este REl, que cristaliza en un proceso de estudio con un grupo de alumnos y un profesor concretos en una institución determinada, constituye implícitamente una respuesta a $\mathrm{Q}_{0}$-FP que puede considerarse surgida de la investigación didáctica. En nuestro caso se trata del REl de los Planes de Ahorro (García, 2005).

Como hemos señalado, cada experimentación y evaluación de un REI sustentado en un MER-S proporciona criterios para contrastar empíricamente el propio MER-S. Esto significa que el análisis a posteriori del desarrollo del REI permitirá comprobar hasta qué punto la actividad matemática que encarna dicho MER-S posibilita alcanzar los objetivos matemático-didácticos previstos de antemano. En todo caso, el citado análisis aportará datos para modificar el MER-S.

Suponiendo que se ha construido un MER-S y que se ha experimentado en Secundaria un REI que lo sustenta, la estrategia metodológica que estamos describiendo propugna utilizar la experiencia y los resultados de dicho proceso para diseñar un REI-FP, cuyo objetivo sea posibilitar el estudio de un problema de la profesión, construido a la vez que el MER-S. La formulación de dicho problema y las primeras etapas de su estudio se llevan a cabo en el Módulo $\mathrm{M}_{0}$, generado por una cuestión $Q_{0}-F P$. La primera tarea que propone la estrategia metodológica para empezar a estudiar dicha cuestión consiste en indagar cuál es la respuesta que aporta la institución escolar y, paralelamente, qué otras posibles respuestas están disponibles en otras instituciones como son la investigación didáctica o la formación del profesorado.

Así, como punto de partida del proceso de formación del profesorado llevado a cabo a lo largo del REI-FP, se planteó la cuestión generatriz $Q_{0}$-FP de dicho REl-FP. Dicha cuestión se propone en el Módulo $M_{0}$ que, en el caso que nos ocupa, se formula, como ya hemos indicado, en los términos siguientes: 
$Q_{0}$-FP: ¿Cómo organizar la enseñanza de la MFE en la ESO y qué papel asignar a la proporcionalidad en dicha organización?

Para facilitar la solución de esta cuestión y ayudar a los profesores en formación a iniciar el trabajo de búsqueda de elementos de respuesta disponibles, se decidió acompañarla con el siguiente conjunto de cuestiones derivadas:

¿Qué características presenta en la ESO la organización matemática curricular en torno a la modelización funcional elemental? ¿Qué tipos de modelos funcionales aparecen? ¿Cómo se relacionan entre sí? ¿A qué cuestiones viene a responder la modelización funcional elemental en la ESO? ¿Por qué y para qué se introducen los modelos funcionales en la ESO? Esto es, ¿̇cuál es la razón de ser oficial que la matemática escolar (el currículo y los libros de texto) asigna a los modelos funcionales que aparecen en la ESO? ¿Qué papel desempeña la proporcionalidad con relación al conjunto de modelos funcionales elementales que aparecen en la ESO?

Además de la respuesta del sistema escolar, ¿qué otras propuestas didácticas alternativas existen (en los trabajos de innovación didáctica, en los artículos de investigación, en los materiales de formación del profesorado, etc.) para organizar la enseñanza de los modelos funcionales en la ESO?

Para buscar respuestas disponibles a estas cuestiones, los profesores en formación se pueden valer de cualquiera de los documentos virtuales y materiales que tengan a su disposición: libros de texto, currículo oficial de la ESO, artículos de investigación e innovación didáctica, materiales de formación del profesorado, producciones de la noosfera, su propia experiencia como alumnos, etc. También cabe la posibilidad de que los formadores indiquen algunos de los media más accesibles, aunque es de suponer que los estudiantes no tengan dificultades en este ámbito.

El diseño a priori de este módulo prevé que la recopilación y análisis preliminar de las respuestas que se aporten a la cuestión $Q_{0}$-FP provocará la emergencia de nuevas cuestiones problemáticas. En particular, la discusión dentro de los grupos de trabajo debe hacer surgir cuestiones relativas al problema de la fundamentación y validación de dichas respuestas y a la propia noción de MFE (ausente en el discurso curricular oficial), así como a las posibles incoherencias internas de las praxeologías matemáticas escolares y a las carencias de componentes praxeológicos que se detectan en los libros de texto. 
Con la realización de esta primera tarea, se pretende que los profesores en formación cuestionen las organizaciones matemáticas escolares en torno a la proporcionalidad y la modelización funcional como punto de partida para analizar las organizaciones didácticas asociadas. Se pretende así superar la tendencia a identificar cualquier ámbito matemático (en este caso la MFE) con los contenidos escolares que aparecen habitualmente en los libros de texto.

Como segunda tarea, se propone que cada grupo exponga brevemente al gran grupo sus conclusiones provisionales relativas a su estudio de la cuestión $Q_{0}$-FP. En gran grupo se discutirán y contrastarán las diferentes respuestas y se elaborará una caracterización provisional de la organización matemática de la ESO en torno a la MFE.

\section{(6) Módulo $M_{1}$ : vivir un REI sustentado en un MER-S}

En este Módulo $M_{1}$ la estrategia metodológica pretende que los profesores en formación empiecen a construir, mediante un trabajo cooperativo, una respuesta a $Q_{0}$-FP. Como primer paso, este módulo constituye un dispositivo didáctico diseñado para proporcionar a los profesores en formación la posibilidad de vivir en propia carne un REI, sustentado en un MER-S y experimentado previamente en Secundaria. Se pretende que los estudiantes construyan por ellos mismos una respuesta a la cuestión generatriz $\mathrm{Q}_{1}$ de dicho REI que contenga, en cierta forma, la proporcionada previamente por los alumnos de Secundaria. Para ello se les propone vivir dicho REI en posición de estudiantes. Esto significa que, en primera instancia, deberán construir una respuesta a $\mathrm{Q}_{1}$ que, en nuestro caso, se materializó en la construcción de una praxeología en torno a los planes de ahorro. Sólo en segunda instancia podrán interpretar, en el Módulo $\mathrm{M}_{2}$, que el trabajo llevado a cabo para construir esta respuesta puede tomarse como una respuesta provisional a $\mathrm{Q}_{0}-\mathrm{FP}$. Esta respuesta, revisable, relativamente análoga a la proporcionada por la TAD (García, 2005), les proporcionará un punto de vista, un sistema de referencia, desde el cual observar, analizar y evaluar otras posibles respuestas a dicha cuestión.

En el REI-FP experimentado, la cuestión generatriz de este módulo -prácticamente idéntica a la experimentada por García (2005)- se formuló como sigue:

$\mathrm{Q}_{1}$ : Deseamos planear con tiempo el viaje de fin de curso, para lo que tenemos que decidir un Plan de Ahorro que nos permita reunir una cantidad suficiente de dinero. Aunque no sabemos aún el precio exacto del viaje, podemos hacer una estimación 
de la cantidad de dinero que necesitamos, y comenzar a tomar decisiones sobre los diferentes plazos de entrega, las diferentes cantidades que deberían aportarse en cada plazo, etc. Por supuesto, no se trata de decidir hoy cuánto dinero hay que entregar ni cómo, sino de empezar a trabajar sobre ello, con la intención de anticiparnos al final de curso y a las necesidades que tendremos cuando sepamos el precio exacto del viaje. El objetivo final es preparar un informe, que podamos presentar a la dirección del centro, y que ayude, en los años sucesivos, a planificar el ahorro de dinero a vuestros compañeros. Este informe debería dar respuesta a cuestiones tales como: ¿Qué posibles planes o estrategias de ahorro se pueden considerar? ¿Qué ventajas e inconvenientes tiene cada uno? ¿Cómo decidir los plazos, las cantidades a dar en cada plazo, la duración del ahorro, etc.?

Como síntesis del trabajo realizado por los grupos y como primer esbozo de la respuesta conjunta de la comunidad de estudio, se elaboró en gran grupo, bajo la dirección de los formadores, un mapa provisional de planes de ahorro que integra y articula las aportaciones de todos los grupos (véase figura 2).

Este mapa constituye el primer esbozo de una praxeología para la enseñanza en torno a la modelización funcional elemental reconstruida por la comunidad de estudio. En dicho mapa aparecen determinadas caracterizaciones de los planes de ahorro (inicialmente mediante la ley recursiva que relaciona cada cuota con la anterior) y, consecuentemente, caracterizaciones de los modelos funcionales asociados a cada tipo de plan de ahorro.

\section{(7) Módulo $M_{2}$ : Analizar el REI vivido sustentado en un MER-S}

En esta etapa de la estrategia metodológica que estamos describiendo, los profesores en formación Ilevan a cabo un análisis matemático-didáctico del REI vivido. Para ello, se retoma el problema de la profesión docente descrito mediante la cuestión $\mathrm{Q}_{0}-\mathrm{FP}$, así como las respuestas parciales que los profesores en formación hayan encontrado en el Módulo $\mathrm{M}_{0}$ a partir de la exploración de los diversos documentos oficiales que tienen a su disposición (incluyendo su propia experiencia como alumnos). Estos datos, junto a una descripción de la respuesta particular a la cuestión $\mathrm{Q}_{0}$-FP surgida en el ámbito de la investigación didáctica y materializada en el REl experimentado en el cuarto curso de la ESO, constituyen los media de los que disponen los profesores en formación para llevar a cabo un análisis matemático-didáctico del REl vivido. 


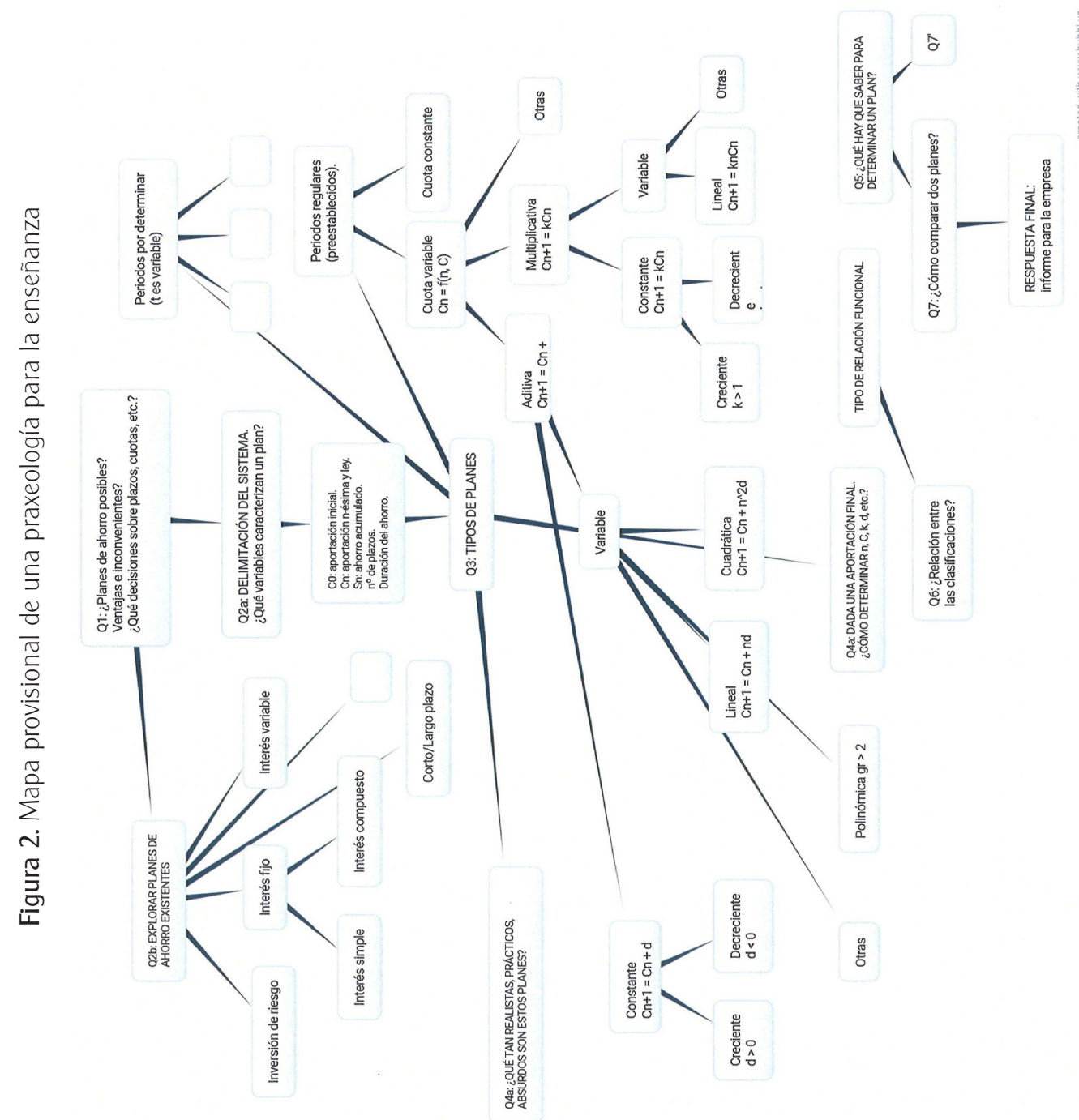


En nuestro caso, las cuestiones planteadas para la realización del análisis mencionado fueron:

$\mathrm{Q}_{21}$ : ¿Cómo se puede describir la actividad matemática desarrollada para responder a la cuestión $\mathrm{Q}_{1}$ ? ¿Cuáles son los elementos matemáticos (nociones, técnicas, propiedades, etc.) utilizados y el procedimiento seguido?

Con el objetivo de precisar el significado de la cuestión $\mathrm{Q}_{21}$ se introdujeron algunas cuestiones derivadas para guiar el análisis praxeológico (matemático) del REI vivido:

¿Se han analizado y comparado las ventajas e inconvenientes de utilizar unas técnicas (aritméticas, algebraicas, gráficas u otras) para estudiar los planes de ahorro?

¿Qué papel han desempeñado las técnicas aritméticas a lo largo del proceso de estudio?

¿En qué momentos concretos del proceso ha surgido la necesidad de utilizar técnicas algebraicas? ¿Qué limitaciones de las técnicas aritméticas se han puesto claramente de manifiesto?

¿Se han articulado las técnicas algebraicas y las técnicas funcionales a fin de abordar ciertas tareas matemáticas?

Para estudiar los planes de ahorro ¿se han utilizado técnicas gráficas? ¿Cómo se han justificado?

¿Qué papel ha desempeñado la proporcionalidad en la actividad matemática desarrollada?

¿Cómo se ha relacionado la proporcionalidad con el resto de relaciones funcionales? ¿Aparece como una relación funcional "especial" o como una más en el conjunto de relaciones funcionales elementales?

¿Consideráis necesario continuar el proceso de estudio varias sesiones más 0 , al contrario, tenéis la sensación de haber dedicado un tiempo suficiente al problema de los planes de ahorro?

¿Cuáles han sido los objetivos del proceso de estudio vivido (aunque de manera incipiente)? Esta cuestión se refiere tanto a los objetivos relativos a los contenidos matemáticos, como a los relativos al tipo de actividad matemática desarrollada.

Por otra parte, se pretendía que los estudiantes comparasen la organización matemática escolar en torno a la modelización funcional -analizada previamentecon la vivida por ellos mismos en el módulo $\mathrm{M}_{1}$. Por lo tanto, las cuestiones que se plantearon (y que enunciamos a continuación) requerían dos respuestas: la relativa al REI vivido y la correspondiente a la actividad matemática escolar habitual, así como la comparación entre ambas. 
¿Se estudian, se analizan, se cuestionan, diferentes tipos de relaciones funcionales que pueden relacionar dos o más magnitudes? ¿Se plantean situaciones en las que se tenga que indagar el tipo de variación que relaciona dos magnitudes o, por el contrario, el tipo de relación funcional está normalmente determinado de antemano?

¿Cuáles son, en cada caso, las cuestiones a las que viene a responder la proporcionalidad? Esto es, ¿̇cuál es la razón de ser que se asigna a la proporcionalidad?

En el caso de la proporcionalidad, ¿̇perviven componentes de la organización clásica como, por ejemplo: razón = cociente entre cantidades de magnitud (que no hay que confundir con fracción = cociente entre dos números enteros) y proporción = igualdad entre dos razones que consta de dos medios y dos extremos? En caso afirmativo, explicad el papel que juegan dichos elementos.

¿Se proponen propiedades de las proporciones (como, por ejemplo, que el producto de medios es igual al producto de extremos) a fin de evitar el uso del instrumento algebraico?

Los problemas de proporcionalidad, ¿̇son considerados como problemas aritméticos o bien como problemas de modelización algebraico-funcional? ¿Qué relación se establece entre el mundo de la proporcionalidad y el mundo funcional?

¿Aparece la regla de tres? En caso afirmativo, ¿se interpreta en términos funcionales o bien en términos aritméticos (meramente numéricos)? ¿Existe en los textos un discurso matemático justificativo de la regla de tres o, por el contrario, ésta aparece como una técnica auto-justificada, esto es, como una técnica que no precisa de ningún tipo de justificación exterior porque es culturalmente inteligible y, como tal, se justifica a sí misma porque "funciona"?

¿Aparecen situaciones problemáticas en las que se pongan claramente de manifiesto las limitaciones de las técnicas aritméticas y la consiguiente necesidad de introducir la herramienta algebraica?

¿Se presenta la proporcionalidad como una relación más, integrada en el ámbito de un conjunto de relaciones funcionales elementales o, por el contrario, aparece aislada?

Las respuestas de los grupos dan lugar a una versión provisional del MER, esto es, del "esqueleto" del REl de los planes de ahorro, que se amplía con la descripción del proceso de estudio en términos de los "gestos de estudio" y de "ayuda al estudio" Ilevados a cabo efectivamente a lo largo del recorrido, tanto por parte de los estudiantes como por parte de los formadores. Para ello los estudiantes utilizaron entre otros media, la documentación relativa a la experimentación llevada a cabo previamente con el REI de los planes de ahorro con alumnos de cuarto curso de ESO (García, 2005). 
La cuestión generatriz de esta fase del segundo módulo se formuló en los términos siguientes:

$Q_{22}$ ¿ ¿Cómo se puede describir la actividad didáctica llevada a cabo en el seno del REI vivido? ¿Qué dispositivos didácticos nuevos han aparecido con relación a la organización didáctica escolar y, en particular, cómo se ha modificado la distribución habitual de las responsabilidades didácticas entre los miembros de la comunidad de estudio?

Y para concretar esta cuestión generatriz y guiar el análisis didáctico del REI vivido se plantearon diez cuestiones derivadas referidas tanto al REI vivido en el módulo $M_{1}$ por los profesores en formación como al experimentado previamente con alumnos de cuarto curso de ESO. Cada cuestión requiere, por lo tanto, dos respuestas así como la comparación entre ellas.

¿Sobre quienes ha recaído, en cada uno de los REl, la responsabilidad de definir y delimitar el sistema de los planes de ahorro?

¿Quién ha tomado, en cada caso, la responsabilidad de elegir el modelo matemático utilizado para estudiar dicho sistema?

¿Qué papel ha desempeñado la cuestión generatriz a lo largo del REl? ¿Se ha mantenido "viva"? ¿Se ha "desvanecido"? ¿Ha permanecido invariante a lo largo del proceso o ha evolucionado?

¿Quiénes se han responsabilizado de plantear las cuestiones y las tareas iniciales? ¿Qué otras tareas podrían haber surgido de dicha cuestión generatriz? ¿Qué otra posible dirección hubiese podido tomar el proceso de estudio?

¿Sobre quiénes ha recaído la responsabilidad de decidir en cada momento los medios, los instrumentos, las técnicas más adecuados para proseguir el estudio?

¿Cómo se ha decidido el tipo de problemas a estudiar en cada momento, así como la dirección que debía tomar el proceso de estudio?

¿Cómo se ha gestionado el tiempo didáctico? Esto es, ¿con base en qué criterios se ha decidido el tiempo que debía dedicarse a cada tipo de problemas?, ¿con base en qué criterios se ha decidido profundizar en un determinado tipo de problemas o, por el contrario, cambiar la actividad para estudiar otro tipo de problemas?

¿Sobre quiénes ha recaído la responsabilidad de evaluar los resultados parciales y las respuestas provisionales que han surgido a lo largo del proceso?

¿Cuál ha sido, en definitiva, el grado de autonomía asumido por los estudiantes a lo largo del REI vivido? 
Uno de los prejuicios más extendidos en el ámbito de la proporcionalidad podría enunciarse como sigue (en forma de "teorema en acto"): "Cuando dos magnitudes se comportan de tal manera que siempre aumentan o disminuyen simultáneamente, entonces son magnitudes directamente proporcionales". ¿Cómo explicar este fenómeno matemático-didáctico?

(8) Construcción de un MER-FPy de la correspondiente praxeología matemática para la enseñanza

A partir de los datos proporcionados por el análisis del REI vivido, surgen cuestiones que extienden y pueden contener ampliamente las que forman parte del MER-S y cuyas respuestas aparecen como necesarias, o al menos útiles, para: (a) redefinir la actividad matemática escolar en torno a la MFE (y, en particular, en torno al papel que se asigna a la proporcionalidad); (b) explicitar la razón de ser oficial que se asigna en la enseñanza secundaria obligatoria a dicho ámbito de la actividad matemática; y (c) interpretar adecuadamente la razón de ser alternativa que el MER-S le asigna y relacionar la MFE así redefinida con los diferentes bloques o áreas de la matemática por enseñar y otros ámbitos de la vida escolar y social.

Éstas son algunas de las cuestiones que estructuran una ampliación y complementan relativamente el MER-S que denominamos MER-FP y que se plantean en esta última etapa de la estrategia metodológica.

En nuestro caso, (véase figura 3) las cuestiones que estructuran el MER-S se limitan esencialmente a las tocantes al tipo de relación funcional entre la cuota k-ésima, $C_{\mathrm{k}}$ o el ahorro acumulado $S_{\mathrm{n}}$ y el número $n$ de imposiciones.

$$
S_{n}=\sum_{k=1}^{k=n} C_{k}
$$

Con el objetivo de precisar el significado de la cuestión $\mathrm{Q}_{21}$ se introdujeron algunas cuestiones derivadas para guiar el análisis praxeológico (matemático) del REI vivido.

En el MER-S las cuestiones se restringen esencialmente a los casos en que las cuotas varían formando una sucesión aritmética o geométrica. En particular, cuando las imposiciones son constantes dan lugar al plan de ahorro equitativo en el que aparece la función de proporcionalidad. 
Como desarrollo de esta problemática elemental, muy rica para alumnos de la ESO, surge el problema de la relación entre ambos tipos de funciones en situaciones mucho más generales.

Dado que en los casos elementales citados $C_{k}$ varía según una relación de recurrencia lineal muy elemental (dada mediante una sucesión aritmética o geométrica), en el MER-FP aparecen, en primera instancia, cuestiones relativas a la relación entre la variación de las cuotas y la variación del ahorro acumulado en el caso en que las imposiciones varían según una ley de recurrencia aditiva, $C_{n+1}=C_{n}+f(n)$, o bien una ley de recurrencia multiplicativa, $C_{n+1}=f(n) \cdot C_{n}$ (donde $f(n)$ es, en principio, una función polinómica). A partir de aquí surge la cuestión no trivial de cómo determinar la evolución de las cuotas (que no tiene por qué ser necesariamente mediante una ley de recurrencia) a partir de una función preestablecida que define la variación del ahorro acumulado, como por ejemplo:

$$
S_{n}=c_{0} \cdot e^{k T} \text { o bien } S_{n}=c_{0}+\sqrt{k T}
$$

Recíprocamente, dada una ley que caracteriza la evolución de las cuotas como, por ejemplo:

$$
\begin{aligned}
& C_{n}=C(1+i) T \cdot n \\
& C_{n+1}=n \cdot \mathrm{k} \cdot C_{n} \\
& C_{n}=C_{n-1}+n^{2} \cdot d
\end{aligned}
$$

o bien

$$
C_{n+2}=C_{n}+C_{n+1}
$$

lo que se propone es determinar la función que describe la evolución del ahorro acumulado.

Esta problemática funcional que estructura el MER-FP y que se materializa en una praxeología matemática para la enseñanza contiene, asimismo, la problemática que hemos denominado del control de los planes de ahorro trabajados o, en el lenguaje de la modelización funcional, la problemática ligada a la construcción de un modelo funcional que cumpla determinadas condiciones (o hipótesis sobre el sistema modelizado) establecidas a priori. También forman parte del MER-FP las cuestiones relativas a la comparación de los planes de ahorro o modelos funcionales en cuestión 


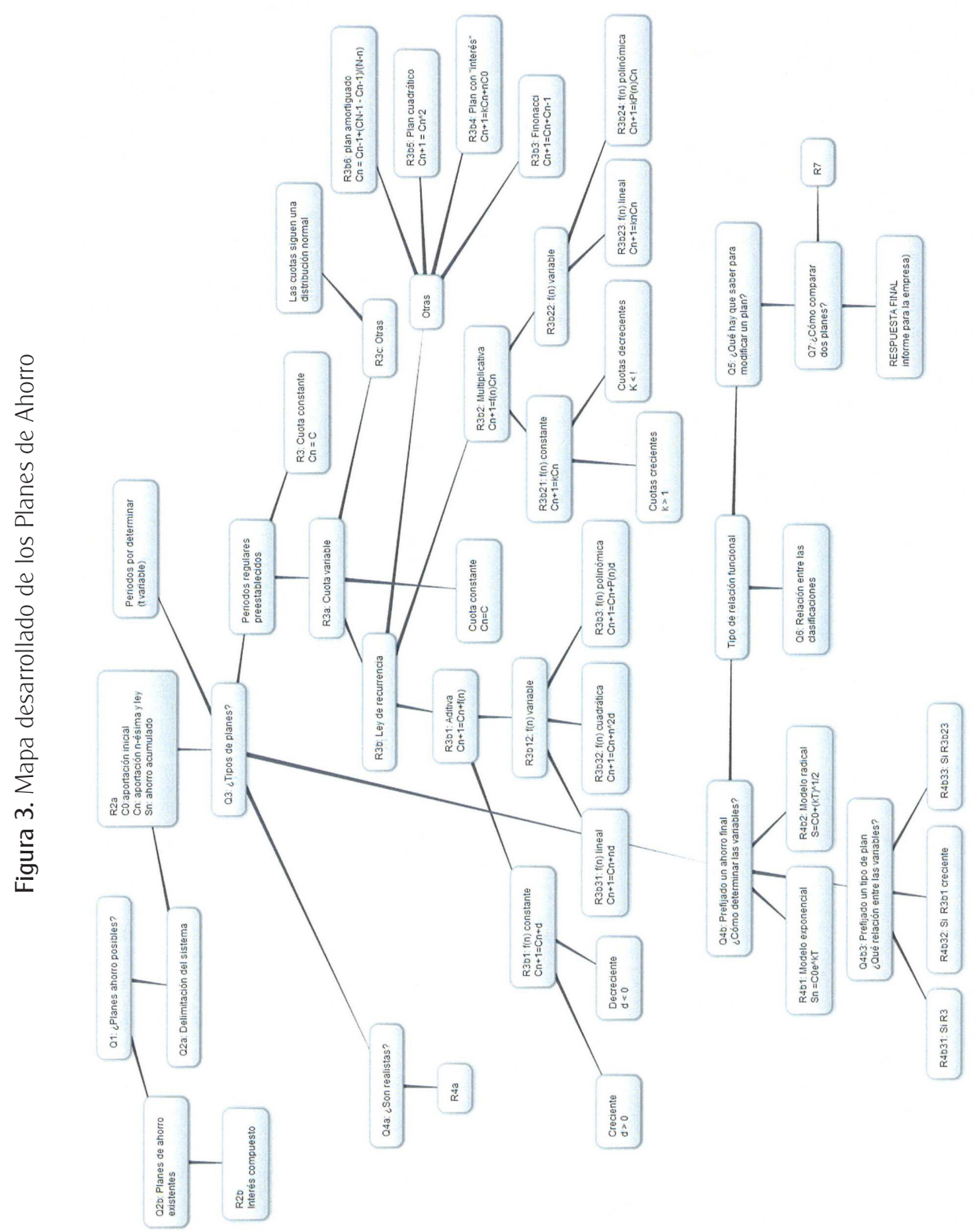




\section{A MODO DE SÍNTESIS: ESTRUCTURA GLOBAL DE UNA ESTRATEGIA PARA LA CONSTRUCCIÓN DE PRAXEOLOGÍAS MATEMÁTICAS PARA LA ENSEÑANZA}

Con el objetivo de mostrar que la estrategia descrita hasta aquí constituye únicamente una parte de una estrategia metodológica más amplia, presentamos un esquema de conjunto (ver figura 4) que contiene ampliamente a la que hemos esquematizado en la figura 1.

Este esquema global sigue conteniendo una primera parte de la estrategia que desemboca en el diseño y experimentación de un REI en Secundaria y que comprende las etapas de (1) a (5). Esta subestrategia tiene sentido en sí misma cuando se trata de construir, simplemente, una praxeología matemática por enseñar (por ejemplo, en Secundaria) sin que ésta tenga que utilizarse necesariamente -aunque pueda utilizarse- como una primera etapa de la estrategia encaminada a construir una praxeología matemática para la enseñanza en el ámbito de la formación del profesorado. Se trata precisamente de la estrategia utilizada en García (2005) y que hemos citado ampliamente.

El esquema global amplía al descrito en la figura 1 mediante una estrategia alternativa completa que no requiere pasar necesariamente por el diseño de una praxeología por enseñar en Secundaria, ni por el diseño a priori de un REI y su consiguiente experimentación. En esta estrategia alternativa las etapas (4), (5), (6), (7) y (8) se reemplazan por las etapas (4'), (6'), (7') (8') y (11).

La principal diferencia entre ambas estrategias consiste en que en esta última se construye directamente un MER-FP (4') con base en ciertos criterios y principios que sustentarían la construcción de un hipotético MER-S (3), pero sin que este MER se haya construido efectivamente y, por tanto, sin basarse en la experimentación previa de un REI sustentado en él. En base al MER-FP se diseña un REl para ser vivido en la institución de Formación del Profesorado (6') y la correspondiente praxeología para la enseñanza (8').

El primer ejemplo que tenemos de la aplicación completa de esta estrategia alternativa se encuentra en Licera (2017). En este trabajo se diseña y se experimenta un MER-FP y se construye la correspondiente praxeología para la enseñanza en torno a los números reales a partir de un esbozo de un MER-S, pero sin basarse en un REI experimentado previamente en Secundaria.

Tenemos, en definitiva, dos estrategias que pueden ser útiles para la construcción de praxeologías matemáticas para la enseñanza y que, en cierto 
sentido, son complementarias. Mientras que la primera descansa en un trabajo previo teórico y experimental en el ámbito de la enseñanza para desembocar en la problemática de la formación del profesorado, la segunda recorre el camino inverso. Pero, en ambos casos, las estrategias pivotan entre la construcción de un fenómeno didáctico en torno a cierto ámbito de la actividad matemática escolar (1) y el estudio de un problema de la profesión docente $\left(1^{\prime}\right)$.

Finalmente, pensamos que la construcción de praxeologías para la enseñanza como un esfuerzo cooperativo entre la investigación didáctica y la institución de formación del profesorado, se podrá consolidar en la medida en que su implementación vaya impregnando la formación de los futuros profesores. 
Figura 4. Esquema de la metodología para construir praxeologías para la enseñanza

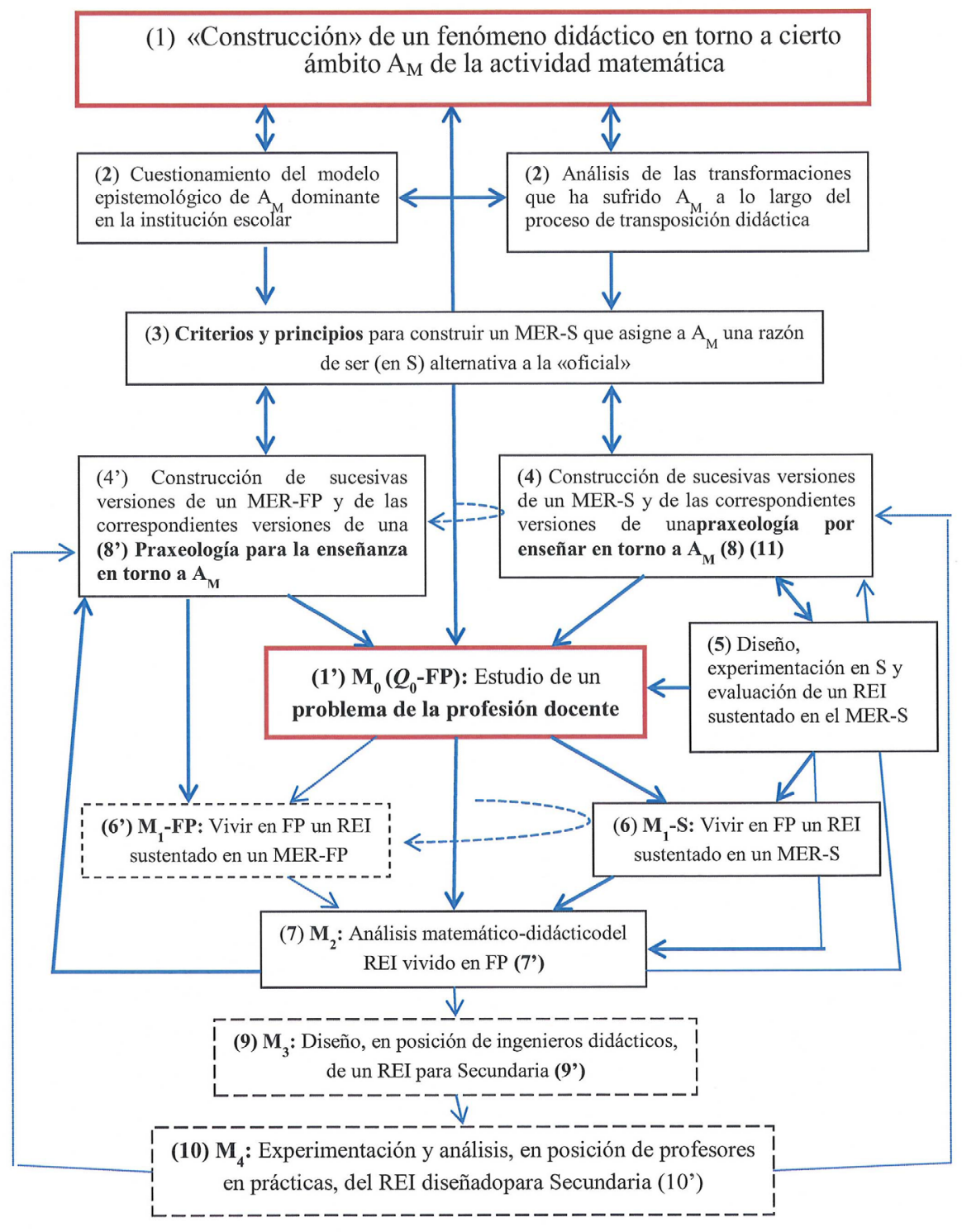




\section{REFERENCIAS}

Arzarello, F., Cusi , A., Garuti, R., Malara, N.A., Martignone, F., Robutti, O., \& Sabena, C. (2013). Meta-didactical transposition: a theoretical model for teachers' education programmes. In A. Clark-Wilson, O. Robutti \& N. Sinclair (Eds.), The Mathematics Teacher in the Digital Era: An international Perspective on Technology Focused Professional Development. (pp. 347-372). Berlin: Springer.

Barquero, B. (2009). Ecología de la modelización matemática en la enseñanza universitaria de las matemáticas. (Tesis de doctorado no publicada). Universitat Autònoma de Barcelona, España.

Barquero, B., Bosch, M. \& Romo, A. (2015). A Study and Research Path on mathematical modelling for teacher education. En K. Krainer \& Vondrová, N. (Eds.), Proceedings of the Ninth Congress of the European Society for Research in Mathematics Education (pp. 809-815). Prague, Czech Republic.

Barquero, B., Bosch, M., \& Romo, A. (2018). Mathematical modelling in teacher education: dealing with institutional constraints. ZDM, 50(1-2), 31-43.

Chevallard, Y. (1985). La transposition didactique. Du savoir savant au savoir enseigné. Grenoble: La Pensée Sauvage.

Chevallard, Y. (1999). Enseignement des mathématiques et besoins professionnels. Le cas des élèves-instituteurs. XVI colloque inter-IREM des PEN et autres formateurs d'instituteurs en mathématiques, Bordeaux. http://yves.chevallard.free.fr

Chevallard, Y. (2003). Didactique et formation des enseignants. Journées d'études INRP-GÉDIAPS. Vingt ans de recherche en didactique de l'Éducation Physique et Sportive à I'INRP (1983-2003). http://yves.chevallard.free.fr

Chevallard, Y. (2013). Enseñar matemáticas en la sociedad de mañana: Alegato a favor de un contraparadigma emergente. Journal of Research in Mathematics Education, 2(2), 161-182.

Cirade, G. (2006). Devenir professeur de mathématiques. Entre problèmes de la profession et formation à I'IUFM (Tesis de doctorado). Université de Provence, Francia.

Florensa, I., Bosch, M., \& Gascón, J. (2016). Lecturer Education: a course design. In 13th International Congress on Mathematical Education. Hamburg.

García, F. J. (2005). La modelización como instrumento de articulación de la matemática escolar. De la proporcionalidad a las relaciones funcionales (Tesis de doctorado no publicada). Universidad de Jaén.

García, F. J., Gascón, J., Ruiz Higueras, L., \& Bosch, M. (2006). Mathematical modelling as a tool for the connection of school mathematics, ZDM The International Journal on Mathematics Education, 38(3), 226-246. 
Gascón, J. (2011). Las tres dimensiones fundamentales de un problema didáctico. El caso del álgebra elemental. Revista Latinoamericana de Investigación en Matemática Educativa, RELIME, 14(2), 203-231.

Licera, M. (2017) Economía y ecología de los números reales en la Enseñanza Secundaria y la Formación del Profesorado, (Tesis de doctorado no publicada), Pontificia Universidad Católica de Valparaíso, Chile.

Ruiz-Munzón, N. (2010). La introducción del álgebra elemental y su desarrollo hacia la modelización funcional (Tesis de doctorado no publicada). Universitat Autònoma de Barcelona, España.

Serrano, L. (2013). La modelización matemática en los estudios universitarios de economía y empresa: análisis ecológico y propuesta didáctica (Tesis de doctorado no publicada). IQS, Universitat Ramon Llull, España.

AliCIA RUIZ Olarría

Dirección: Dpto. Didácticas Especiicas

Facultad de Formación de Profesorado y Educación

Universidad Autónoma de Madrid

28049 Madrid, España

Teléfono: +34914974798 